\title{
Microfibrillar buckling within fibers under compression
}

\author{
Theo Odijk \\ Faculty of Chemical Engineering and Materials Science, Delft University of Technology, P.O. Box 5045, \\ 2600 GA Delft, The Netherlands
}

(Received 13 November 1997; accepted 22 January 1998)

\begin{abstract}
A tentative theory is presented of microfibrillar buckling within compressed fibers. A quantitative harmonic analysis is given of the semiclassical buckling of a clamped stiff chain; the influence of thermal undulations is incorporated in Euler buckling. A scaling analysis including entropy allows one to understand semiclassical buckling. The buckling of a microfibril within a fibrous environment is analyzed in two limits: (a) when the fiber is incompressible; (b) when the matrix is assumed to be a fixed harmonic potential. In the latter case, a network of microfibrils may melt at high enough compression before the usual buckling occurs. We also study the renormalization of the confining potential by long-range elastic fields. A provisional comparison with experimental studies on macroscopic failure is given. C) 1998 American Institute of Physics. [S0021-9606(98)51416-X]
\end{abstract}

\section{INTRODUCTION}

A complete theory of the compressive failure of liquidcrystalline fibers will have to settle several key issues. Ideally, the fibrillar morphology obtained by coagulation and heat treatment would have to be predicted a priori. If not, detailed information concerning the fibrillar structure would have to be gathered by various experimental techniques. ${ }^{1}$ Second, the fibrils are supposed to behave as separate entities within the surrounding fibrillar matrix; the fiber cannot be regarded or modeled as a homogeneous elastic medium. It seems plausible that, upon deforming a fiber, an individual fibril or microfibril may slide with respect to its confining environment, at least to some extent. The interaction between filament and environment may remain substantial nonetheless. ${ }^{2,3}$ Theoretically, then, a treatment combining fibrillar statistics and elasticity could be devised at the single fibril or microfibril level. One difficulty would be their noncylindrical form. ${ }^{1}$ Yet microfibrils are also thin enough ${ }^{4}$ to be perturbed strongly by thermal motion. Moreover, since the microfibrils apparently play a predominant role in certain cases of compressive failure of macroscopic fibers, ${ }^{4}$ a theory of failure ought to have a basis in statistical mechanics. Finally, given the fact that fiber buckling is a nonlinear and possibly dynamic phenomenon, the formulation of a quantitative theory is a formidable undertaking by any standard.

An obviously simplified approach to the physics of fiber compression emphasizing microfibrillar buckling is presented here. It is analogous to "fiber crushing" discussed in the literature on composite materials. ${ }^{5}$ It is hoped that our statistical theory will be of some qualitative use in gaining insight into macroscopic failure. Fortunately, there seem to be some empirical grounds ${ }^{4}$ for the hypotheses employed here. Since thermal motion may well be important, I need to investigate what I shall term the "semiclassical" buckling of a single microfibril encapsulated in an effectively elastic environment. The "classical" case corresponds to a theory based on the usual Euler buckling; ${ }^{6}$ the term "semiclassical" refers to the influence of thermal motion on elasticity theory ${ }^{7,8}$ and alludes to semiclassical approximations similar to those encountered in quantum mechanics at relatively short de Broglie wavelengths. Furthermore, I give merely an analysis valid for weak undulations of the (cylindrical) microfibril; a full analysis should be nonlinear. The same model could be of use in problems involving the buckling of biofilaments within a complex cellular environment. ${ }^{9-11}$

Before discussing the compressional failure of a microfibril within a fiber, I need to describe the semiclassical buckling of a single stiff chain for which no theory exists. ${ }^{12}$ An analytical theory is developed together with a scaling interpretation and this is juxtaposed against classical Euler buckling. ${ }^{6}$

\section{SEMICLASSICAL BUCKLING OF A STIFF CHAIN}

\section{A. Euler buckling}

A stiff chain may be viewed as an ideally elastic rod of contour length $l$. Its configuration is completely characterized by the unit vector $\mathbf{u}(s)$ tangential to the chain at contour distance $s$ from one end. The Hamiltonian of the rod under a compressive force $\mathbf{f}$ is

$$
\mathscr{B}=\frac{1}{2} P k_{B} T \int_{0}^{l} d s\left(\frac{\partial \mathbf{u}}{\partial s}\right)^{2}+\int_{0}^{l} d s \mathbf{u} \cdot \mathbf{f}
$$

[see Fig. 1(a)]. The bending force constant ${ }^{13}$ is here written as the product of the persistence length $P$ and the temperature $T\left(k_{B}=\right.$ Boltzmann's constant $)$. The material deformation ${ }^{7}$ may be neglected at compressions right up to buckling. Even though $\mathscr{H}$ is purely elastic, it is anharmonic because of the local constraint ${ }^{13} \mathbf{u}^{2}(s)=1$ for all $s(0 \leqslant s \leqslant l)$. Hence, a buckling theory for a rod restricted under various conditions (e.g., clamped positions and angles at the two ends) would appear to require a nonlinear analysis. Still, it is well known that Euler buckling may be evaluated exactly by dealing with the limit of solely weak undulations.

We turn to the harmonic approximation $\mathscr{H}_{h}$ to Eq. (1) which is valid for weak fluctuations away from the rod limit. The compressive force $\mathbf{f}$ points in the $z$ direction of our Car- 


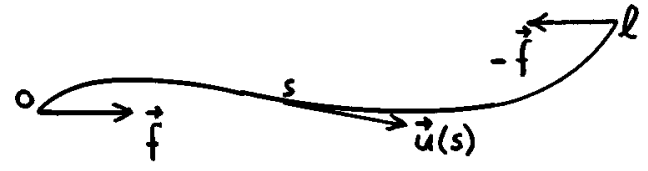

(a)

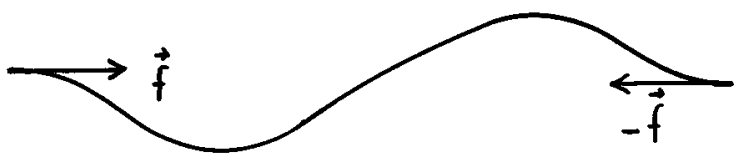

(b)

FIG. 1. (a) An elastic rod in a certain configuration $\mathbf{u}(s)$ under compression; (b) with ends orientationally fixed along the direction of the compressive force.

tesian coordinate system $(\mathbf{r}=x, y, z)$; the undulations are weak so we write $\mathbf{u}(s) \simeq\left(\theta_{x}(s), \theta_{y}(s), 1\right) \equiv(\boldsymbol{\theta}(s), 1)$ correct to the linear order in the small angles $\theta_{x}(s)$ and $\theta_{y}(s)$ of the vector $\mathbf{u}(s)$ with respect to $\mathbf{f}$

$$
\begin{aligned}
& \left(\theta_{x}(s) \ll 1 ; \quad \theta_{y}(s) \ll 1\right), \\
& \mathscr{H}_{h}=\frac{1}{2} P k_{B} T \int_{0}^{l} d s\left(\frac{d \boldsymbol{\theta}}{d s}\right)^{2}-\frac{1}{2} f \int_{0}^{l} d s \boldsymbol{\theta}^{2} .
\end{aligned}
$$

I next discuss Euler buckling ${ }^{6}$ under restraints useful for the purposes at hand. Formally, we would let the temperature go to zero; in practice we let the product $P T$ remain constant and let the ratio $l / P$ become as minute as possible; for a macroscopic rod it is easy to attain $l / P<10^{-15}$ at room temperature. A simple configuration not without experimental interest ${ }^{9}$ is when the two ends of the rod or filament are clamped at fixed orientations $[\boldsymbol{\theta}(o)=0 ; \boldsymbol{\theta}(l)=0$; see Fig. 1(b)]. The end positions defined within a plane perpendicular to $\mathbf{f}$ are unconstrained. Note that within the harmonic approximation, the undulations in the $x$ and $y$ directions are separable.

In the $x$ direction, the Euler-Lagrange equation associated with Eq. (2) is

$$
P k_{B} T \frac{d^{2} \theta_{x}}{d s^{2}}+f \theta_{x}=0 .
$$

One solution is $\theta_{x, 1}=0$ but a second solution,

$$
\theta_{x, 2}=A \sin \pi s / l,
$$

appears when $f$ attains the value given by

$$
f_{c}=\frac{\pi^{2} P k_{B} T}{l^{2}} .
$$

The constant $A$ cannot be specified at the harmonic level. The critical force $f_{c}$ signifies the compression under which the rod buckles since the state with $\theta_{x, 1}=0$ becomes unstable whenever $f>f_{c}$. A fully nonlinear theory would yield a second solution whose form is similar to that of Eq. (4) with an amplitude $A=\mathscr{O}(1)$.

\section{B. Semiclassical buckling}

I now study the effect of "nonzero temperatures," i.e., $l / P$ is no longer vanishingly small. This applies to filaments of mesoscopic dimensions. We need to analyze the properties of the appropriate partition or Green function $G$ of a compressed wormlike chain; the elastic rod of the previous section is now immersed in a heat bath of temperature $T$. Again we would like a rigorous theory taking into account the full nonlinearity of the Hamiltonian Eq. (1). But it suffices here to compute $G$ within the harmonic approximation based on Eq. (2).

Let the filament first be constrained at its two ends as follows: $\boldsymbol{\theta}(0)=\boldsymbol{\theta}_{1}$ and $\boldsymbol{\theta}(l)=\boldsymbol{\theta}_{2}$. Now if $\mathbf{f}$ were negative it would be an extensional force. Then, Eq. (2) would be the Hamiltonian of a wormlike chain in a strong tensile or nematic field with nematic coupling parameter $\Gamma \equiv-f P / k_{B} T$ $\equiv \beta_{t}^{2}$. This Hamiltonian is equivalent to that of a quantum harmonic oscillator as noted a long time ago. ${ }^{14}$ The appropriate Green function has been analyzed in detail, ${ }^{14}$

$$
\begin{aligned}
G_{t}\left(\boldsymbol{\theta}_{1}, \boldsymbol{\theta}_{2} ; l\right)= & \frac{\beta_{t}}{2 \pi \sinh N \beta_{t}} \exp -\frac{\beta_{t}}{2 \sinh N \beta_{t}}\left[\left(\boldsymbol{\theta}_{1}^{2}+\boldsymbol{\theta}_{2}^{2}\right)\right. \\
& \left.\times \cosh N \beta_{t}-2 \boldsymbol{\theta}_{1} \cdot \boldsymbol{\theta}_{2}\right] \quad(f<0)
\end{aligned}
$$

with $N \equiv l / P$. Analytic continuation by inverting the sign of the force immediately yields the partition function for a stiff chain under compression,

$$
\begin{aligned}
G_{c}\left(\boldsymbol{\theta}_{1}, \boldsymbol{\theta}_{2} ; l\right)= & \frac{\beta_{c}}{2 \pi \sin N \beta_{c}} \exp -\frac{\beta_{c}}{2 \sin N \beta_{c}}\left[\left(\boldsymbol{\theta}_{1}^{2}+\boldsymbol{\theta}_{2}^{2}\right)\right. \\
& \left.\times \cos N \beta_{c}-2 \boldsymbol{\theta}_{1} \cdot \boldsymbol{\theta}_{2}\right] .
\end{aligned}
$$

(Compressive coupling parameter $\Gamma_{c} \equiv f P / k_{B} T \equiv \beta_{c}^{2} ; f>0$.) If the chain is clamped as in the previous section $[\boldsymbol{\theta}(o)$ $=0 ; \boldsymbol{\theta}(l)=0]$, the free energy of the filament under compression is simply

$$
\begin{aligned}
F & =-k_{B} T \ln G_{c}(o, o ; l) \\
& =k_{B} T\left(\ln \sin N \beta_{c}+\ln 2 \pi-\ln \beta_{c}\right) .
\end{aligned}
$$

The distribution of the angle $\boldsymbol{\theta}$ at contour position $m$ is Gaussian,

$$
\begin{aligned}
P(\boldsymbol{\theta}, m) \equiv & \frac{G_{c}(o, \boldsymbol{\theta} ; m) G_{c}(\boldsymbol{\theta}, o ; l-m)}{G_{c}(o, o ; l)} \\
= & \frac{\beta_{c} \sin \left(\beta_{c} l / P\right)}{2 \pi \sin \left(\beta_{c} m / P\right) \sin \left(\beta_{c}(l-m) / P\right)} \exp -\frac{\beta_{c} \theta^{2}}{2} \\
& \times\left[\operatorname{cotg}\left(\beta_{c} m / P\right)+\operatorname{cotg}\left(\beta_{c}(l-m) / P\right] .\right.
\end{aligned}
$$

The second moment is given by

$$
\left\langle\theta^{2}(m)\right\rangle=\frac{2 \sin \left(\beta_{c} m / P\right) \sin \left(\beta_{c}(l-m) / P\right)}{\beta_{c} \sin \left(\beta_{c} l / P\right)} .
$$

In particular, the mean-square undulation at the midpoint is

$$
\left\langle\theta^{2}\left(\frac{1}{2} l\right)\right\rangle=\beta_{c}^{-1} \operatorname{tg}\left(\beta_{c} l / 2 P\right) .
$$

At this juncture we have to exercise caution in interpreting Eqs. (7)-(11). At zero compression $\left(f \rightarrow 0\right.$ or $\left.\beta_{c} \rightarrow 0\right)$, the second moment at the midpoint reduces to 


$$
\left\langle\theta^{2}\left(\frac{1}{2} l\right)\right\rangle=\frac{l}{2 P} \quad(l \ll P) .
$$

This is what one expects on the basis of the usual Gaussian statistics for the undulations of a short wormlike chain. As the compressive force is enhanced, the undulations increase gradually [Eq. (11)]. However, a strong increase in the fluctuations is predicted to occur as $\beta_{c}$ approaches $\pi P / l$. In order to understand this effect, we Taylor expand the tg term

$$
\left\langle\theta^{2}\left(\frac{1}{2} l\right)\right\rangle \simeq\left(\frac{k_{B} T}{f P}\right)^{1 / 2}\left[\frac{2}{\pi-\left(l^{2} f / P k_{B} T\right)^{1 / 2}}\right] .
$$

At a certain compression $f_{1}$, the undulations become so marked that $\left\langle\theta^{2}\left(\frac{1}{2} l\right)\right\rangle=\mathscr{O}(1)$,

$$
f_{1}=f_{c}\left(1-\frac{c_{1} l}{P}\right) \text {. }
$$

Here, $f_{c}$ is the classical compression given by Eq. (5). In deriving Eq. (14), Eq. (13) has been extended to its outer limit of validity where anharmonic terms in the context of Eq. (2) start to exert themselves. Accordingly, we cannot ascertain the exact value of the numerical constant $c_{1}$ which is of order unity nevertheless.

The meaning of Eq. (14) is as follows, The semiclassical variant of the first classical solution $\boldsymbol{\theta}_{1}=0$ is the distribution given by Eq. (9). At large enough compressions there exists a semiclassical variant of the second classical solution with $\boldsymbol{\theta}_{2}(s)$ approximated by Eq. (4) and with $A=\mathscr{O}(1)$. The latter variant has not been computed here. It would entail a difficult nonlinear analysis similar to those developed in other cases. ${ }^{8,15}$ Now, at a compression $f_{1}$ the two distributions would be indistinguishable since the respective amplitudes of the undulations are the same. Buckling must therefore occur at a somewhat lower compression $f_{s}$,

$$
f_{s}=f_{c}\left(1-\frac{c_{s} l}{P}\right)
$$

where $c_{s}>c_{1}$ but we insist on $c_{s}=\mathscr{Q}(1)$ in view of the structure of Eq. (13). For macroscopic rods we obviously regain the classical limit. Note that Eq. (15) predicts the obviation of the buckling transition for long enough chains ( $l$ $\left.\geqslant P / c_{s}\right)$.

It has been shown that a relevant scale for semiclassical buckling is the persistence length $P$. The precise condition for the onset or absence of a buckling transition will have to be determined by a complete anharmonic theory. It stands to reason that a family of expressions like Eq. (15) exists depending on the various clamping conditions on the ends of the semiflexible filament. Experimentally, it would be interesting to monitor the strong increase in undulations near the buckling transition with the help of scattering or microscopy techniques.

\section{Scaling picture}

For small contour lengths $\left(l<\frac{1}{2} P\right)$, the semiclassical limit is close to classical Euler buckling. It is reasonable that globally deformed configurations for compressed worms are similar in form to those described by Eq. (4) (but note that we are now focusing on thermally induced deviations from the solution of the first kind and the amplitude $A \ll 1$ ). Thus, the chain may be regarded essentially as a single fluctuating sigmoidal curve of root-mean-square amplitude $\psi$ $\equiv\left\langle\theta^{2}\left(\frac{1}{2} l\right)\right\rangle^{1 / 2}$. This bend in turn behaves more or less as two connected rods confined within their two respective conical sections so that the free energy of entropic confinement should be given by

$$
F_{\text {conf }} \simeq-k_{B} T \ln \psi^{2} \text {. }
$$

Next, there is a bending energy $F_{b}$ and a compression energy $F_{\text {comp }}$ which are estimated from Eq. (2),

$$
\begin{aligned}
& F_{b} \simeq P l^{-1} \psi^{2} k_{B} T, \\
& F_{\mathrm{comp}} \simeq-f l \psi^{2} .
\end{aligned}
$$

Upon minimizing the total free energy, the sum of Eqs. (16) -(18), with respect to $\psi$, we get

$$
\psi^{2}=\left\langle\theta^{2}\left(\frac{1}{2} l\right)\right\rangle \simeq \frac{1}{(P / l)-\left(f l / k_{B} T\right)} .
$$

It is easy to check that this form is basically equivalent to Eq. (11) if we take into due consideration the uncertainty in the numerical coefficients. We have therefore established that the confinement entropy indeed arises in the way anticipated above. Equation (16) may be used to formulate convenient qualitative anlayses in the semiclassical limit in those cases when it would be tedious to set up precise statistical theories. As usual, numerical coefficients are often disregarded within scaling approximations.

\section{BUCKLING OF MICROFIBRILS WITHIN FIBERS}

We investigate two extremes that are readily analyzed. Within scaling theory, the type of clamping-positional or orientational-need not be specified for it merely affects the values of the numerical coefficients.

\section{A. Incompressible limit}

Suppose the typical spacing $\Delta$ between a test microfibril and its surrounding environment of fibrillar material is invariant because the fiber itself is considered incompressible in the two directions transverse to the compressive force. The fibers are unyielding beyond a distance $\Delta$. For instance, the fiber could be clamped within a metal cylinder. The deflection length ${ }^{16}$ of the microfibril is given in terms of its persistence length $P$,

$$
\lambda_{0}=\Delta^{2 / 3} P^{1 / 3} \text {. }
$$

It represents the distance the microfibril wanders before coming into contact with the fibrillar matrix again. The undulations are caused by thermal motion. The deflection length is independent of the compressive force under these circumstances. For a close-packed fiber where $\Delta$ is typically only $0.1 \mathrm{~nm}$ wide, the deflection length is often much smaller than the length $l$ of the microfibril. Hence, we focus on the important regime of strong undulations $\left(l \gg \lambda_{0}\right)$ and we neglect end effects.

We next add to Eq. (2) a harmonic confining potential of the form, 


$$
U_{\mathrm{ext}}=\frac{k_{B} T}{2 B^{3}} \int_{0}^{l} d s\left(x^{2}+y^{2}\right),
$$

which simulates the environment surrounding the microfibril. Here, $x=x(s)$ and $y=y(s)$ denote the transverse position of point $s$ on the microfibrillar contour. The variable $B$ of dimension length is to be regarded as adjustable because incompressibility of the fiber must be imposed at the end of the computation. A harmonic potential like Eq. (21) has been used in previous investigations of the buckling of fibrous composites in the purely classical limit. ${ }^{5,17}$ The statistical mechanics of the total Hamiltonian $\mathscr{H}_{h}+U_{\text {ext }}$, but with $f$ $<0$, has been discussed recently in polymer physics. ${ }^{18}$ Nonetheless, I wish to analyze microfibrillar buckling in a qualitative way only in view of the approximations inherent in the model.

A fast way of deriving results is to note that a fully harmonic Hamiltonian implies a Gaussian distribution $\mathscr{D}$ of infinitesimal segments belonging to the microfibril,

$$
\mathscr{D} \sim \exp -\left(x^{2}+y^{2}\right) / d^{2} .
$$

Here, the width $d$ is momentarily assumed to be a variational parameter. The deflection length $\lambda(d)=d^{2 / 3} P^{1 / 3}$ would imply a typical angle $\psi \simeq d / \lambda$ of a deflection segment with respect to the director. The total free energy of the microfibril is then estimated to be

$$
F_{\text {tot }} / k_{B} T \simeq \frac{l}{\lambda}-\frac{f l d^{2}}{k_{B} T \lambda^{2}}+\frac{d^{2} l}{B^{3}},
$$

where $f$ is the compressive force on the microfibril. The first term is the confinement free energy which must be extensive and proportional to the microfibrillar length. ${ }^{14,16}$ Minimizing $F_{\text {tot }}$ with respect to $d$, we obtain

$$
\frac{d^{2}}{B^{3}} \simeq \frac{1}{\lambda}+\frac{f d^{2}}{k_{B} T \lambda^{2}} .
$$

Now in view of the incompressibility constraint, $d$ must be set equal to $\Delta$. This imposes the following value on the harmonic parameter $B$,

$$
B^{3} \simeq \frac{\Delta^{2} \lambda_{0}}{l+\frac{f \Delta^{2}}{k_{B} T \lambda_{0}}} .
$$

As we exert a greater compressive force on the fiber, the effective harmonic potential confining a microfibril also has to increase so as to render the fiber incompressible as a whole. Very little happens with regard to the undulations, though this analysis is of methodological interest. Much more goes on when we consider the opposite extreme.

\section{B. Fixed harmonic potential}

We now postulate a fixed potential, $v$ per unit length of microfibril, exerted on it by the fibrillar background. This is approximated by a harmonic well

$$
U_{h} / k_{B} T=\frac{1}{2} w_{0} \int d s\left(x^{2}+y^{2}\right) .
$$

In the ideal case, the coefficient $w_{0}$ is the second derivative of $v$, scaled by $k_{B} T$, with respect to the transverse vector. However, a deformation of the microfibril induces a longrange elastic field in the surrounding material; the coefficient $w_{0}$ may have to be renormalized (see the Appendix for details).

As remarked above, the length of the microfibril will often be greater than its deflection length $\lambda_{0}$ when the fiber is in the initial, unstressed state. Following the procedure above for computing undulations, we get

$$
F_{\text {tot }} / k_{B} T \simeq \frac{l}{\lambda}-\frac{f l d^{2}}{k_{B} T \lambda^{2}}+w_{0} d^{2} l \quad\left(\lambda=d^{2 / 3} P^{1 / 3}\right) .
$$

Minimization of this expression with respect to $d$ yields

$$
w_{0} d^{2} \simeq \lambda^{-1}\left(1+\frac{f d^{2}}{k_{B} T \lambda}\right) .
$$

Initially, at zero compression $(f=0)$, the undulatory amplitude is given by

$$
w_{0} d_{0}^{8 / 3} P^{1 / 3} \simeq 1 \quad\left(\lambda_{0}=d_{0}^{2 / 3} P^{1 / 3} \ll l\right) .
$$

Since $w_{0}$ is constant, exerting a compressive force on the fiber will gradually enhance the undulations according to Eq. (28).

Let us next suppose microfibrils of the same type constitute the overwhelming majority of filaments within the fibrous material. The microfibrils need not be monodisperse with regard to contour length. The fiber may then be roughly envisaged as a positionally ordered system of microfibrils. At high enough compressions, this may well melt into a lessordered state, the melting transition being given by a Lindemann criterion ${ }^{18}$

$$
\frac{d}{R}=c_{l}
$$

The average distance between the microfibrils is $R$ and $C_{l} \simeq 0.15$ is a constant. According to Eqs. (28) and (30), the microfibrillar network could melt at a certain $f_{m}$, clearly implying compressive failure of the macroscopic fiber.

We need to bear in mind that Lindemann melting may be pre-empted as ultimately the deflection length could become as large as $l$ while $f<f_{m}$. Equation (28) will break down at a characteristic compression $f_{l}$,

$$
w_{0} l^{2} \simeq f_{l} / k_{B} T \quad\left(\lambda_{l}=d_{l}^{2 / 3} P^{1 / 3}=l\right) .
$$

For $f>f_{l}$, we enter a new regime described by semiclassical buckling. Then, the microfibril may be viewed as a single fluctuating bend of typical width $\left(x^{2}\left(\frac{1}{2} l\right)+y^{2}\left(\frac{1}{2} l\right)\right)^{1 / 2}$ $\simeq \psi l$. The total free energy is the sum of a bending energy, energy of compression, entropy, and harmonic energy,

$$
F_{\text {tot }} / k_{B} T \simeq \frac{P \psi^{2}}{l}-\frac{f \psi^{2} l}{k_{B} T}-\ln \psi^{2}+w_{0} l^{3} \psi^{2} .
$$

Minimization of $F_{\text {tot }}$ with respect to $\psi$ leads to

$$
\psi^{2}\left(w_{0} l^{3}+P l^{-1}-\frac{f l}{k_{B} T}\right) \simeq 1
$$




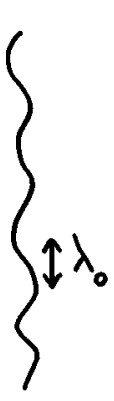

(i)

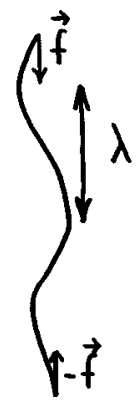

(ii)

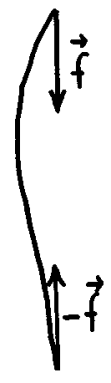

(iii)
FIG. 2. A microfibril confined within a fiber and under a compressive force f. (i) Initial state $(\mathbf{f}=0)$; undulations are present with a deflection length $\lambda_{0}$ [see Eq. (29)]; (ii) undulations are gradually forced out by a compressive force; $d$ and $\lambda$ are given by Eq. (28). Melting of a microfibrillar lattice may occur if a Lindemann criterion [Eq. (30) is met]; (iii) only one undulation remains at high enough compressions $\left(f \geqslant f_{l}\right)$. Angular deviations are given by Eq. (33). Ultimately, compressive failure of the microfibril occurs at a critical force expressed by Eq. (34).

This expresses the angular deviation as a function of the compressive force $f$

Next, we know that $w_{0} l^{4} \gg P$ since $w_{0} d_{l}^{4} \lambda_{l} \gg 1$, and moreover $P \gg l$ in any event. Equation (33) reduces to a very simple classical limit for microfibrillar failure,

$$
f_{c} \simeq w_{0} l^{2} k_{B} T \text {. }
$$

The sequence of events described by Eqs. (28)-(34) is illustrated in Fig. 2. We do not know the coefficients in Eqs. (31) and (34) within a limited scaling theory. Whether or not there is a regime $f_{l}<f<f_{c}$, remains to be seen. Yet a microfibril of length $l$ does buckle as the compression reaches the limit defined by Eq. (34); undulations have been forced out of the state the microfibril was in originally. If the fiber can be approximated as a network of basically monodisperse microfibrils, Eq. (34) would also express the onset of macroscopic failure.

There is one case where $w_{0}$ may be readily estimated. Let the fiber be a compact network of microfibrils of length $l$. The microfibrils are held together by junctures at their ends and their interaction is hard-core. The energy scale per microfibril is then pinned by the thermal energy so that $v$ $\simeq k_{B} T / l$. If $\Delta$ is a scale characterizing allowable transverse undulations, we have $v^{\prime \prime} \simeq k_{B} T / l \Delta^{2}$. The critical force is then given by

$$
f_{c} \simeq \frac{l k_{B} T}{\Delta^{2}}
$$

\section{CONCLUDING REMARKS}

We tentatively compare the predictions derived above with experiment, on the assumption that microfibrillar buckling expressed by Eq. (35) indeed reflects the onset of compressive failure at the macrolevel. The compressive strength of liquid-crystalline polyaramide (PBT) fibers ${ }^{4}$ is about 0.3 $\mathrm{GPa}$, valid presumably at room temperature [stronger polyaramide (PPT) fibers have been manufactured recently $\left.{ }^{19}\right]$. The PBT microfibrils in the as-spun state as evidenced in electron micrographs, appear to be $10 \mathrm{~nm}$ wide and $100 \mathrm{~nm}$
$\operatorname{long}^{4}(D=10 \mathrm{~nm}, l=100 \mathrm{~nm})$. The compressive yield would be $\sigma \simeq f_{c} / D^{2}$ with $f_{c}$ given by Eq. (35). This would imply a transverse scale $\Delta$ of about $0.1 \mathrm{~nm}$ which seems entirely reasonable for a densely packed fiber. Ideal buckling expressed by Eqs. (5) and (14) certainly has nothing to do with the failure of the fiber for in that case $\sigma<1 \mathrm{MPa}$ if the persistence length is estimated as $10^{4} \mathrm{~nm}$. It is remarked that the deflection length $\left(\lambda=\Delta^{2 / 3} P^{1 / 3}=4 \mathrm{~nm}\right)$ is much smaller than the microfibrillar length $l$ although Lindemann melting would not occur. Under the experimental conditions, the undulatory amplitude $d$ as predicted in Eq. (28) is simply too small.

In conclusion, we have formulated a mesoscopic theory of microfibrillar buckling at the harmonic level. The precise range of validity of such mesoscopic analyses remains to be seen of course. In a related problem-the melting of closepacked hexagonal DNA crystals under zero stressundulation theory ${ }^{20}$ agrees well with experiment. ${ }^{21}$ Computer simulations of fibers modeled at the atomic level seem a long way off. For the sake of consistency, the quenched state would have to be simulated a priori and this is probably quite difficult to achieve.

\section{APPENDIX}

The coefficient $w_{0}$ may need to be renormalized by the elastic fluctuations of the effective medium surrounding the test microfibril. Let us consider the material to be isotropic in a rough zeroth-order approximation. Its behavior is supposed to be rubberlike so its Poisson's ratio is close to half. The vector $\boldsymbol{\epsilon}(\mathbf{r})$ signifying the displacement of the material from equilibrium satisfies a Laplace equation ${ }^{6}$

$$
\Delta \boldsymbol{\epsilon}(\mathbf{r}) \simeq 0 .
$$

We focus on the elastic energy of the test microfibril together with that of the deformed surroundings,

$$
\begin{aligned}
& U_{\mathrm{el}}=\frac{1}{2} w_{0} k_{B} T \int_{0}^{l} d s(\mathbf{u}(s)-\boldsymbol{\epsilon}[\mathbf{r}(s)])^{2}+\frac{1}{2} K k_{B} T \int d \mathbf{r}(\boldsymbol{\nabla} \\
& \cdot \boldsymbol{\epsilon})^{2} .
\end{aligned}
$$

This states that the medium responds elastically to a deformation of the microfibril. In view of Eq. (A1), the response has a long range. A detailed statistical model would be needed to compute the bulk modulus $K$.

At this stage it is well to note that Eqs. (A1) and (A2) are precisely isomorphous to those pertaining to a test chain enclosed within a nematic matrix as discussed by de Gennes. ${ }^{22}$ Thus we simply quote his end result for the renormalized coefficient $w$. For a chain, or a microfibril of radius $a=\frac{1}{2} D$ in our case, deformed at a wave vector $q$, the original $w_{0}$ is transformed into a renormalized $w$,

$$
\begin{aligned}
& w^{-1}=w_{0}^{-1}+w_{1}^{-1}, \\
& w_{1}=2 \pi K /-\ln q a .
\end{aligned}
$$

The meaning of Eq. (A3) is that the deformed chain and its response by the surrounding medium work in series with regard to the compliances $w_{0}^{-1}$ and $w_{1}^{-1}$. In case the undulations are strong, we obviously have $q \simeq 2 \pi / \lambda$, whereas $q$ 
$\simeq 2 \pi / l$ in the semiclassical regime. Hence it is possible to estimate $w_{1}$ and then use $w$ instead of $w_{0}$ in the body of the paper. In this way, we need not worry further about the long range of the elastic response.

${ }^{1}$ L. C. Sawyer, R. T. Chen, M. G. Jamieson, I. H. Musselman, and P. E. Russell, J. Mater. Sci. 28, 225 (1993).

${ }^{2}$ J. H. Greenwood and P. G. Rose, J. Mater. Sci. 9, 1809 (1974).

${ }^{3}$ D. I. Green, A. P. Unwin, and G. R. Davies, and I. M. Ward, Polymer 31, 579 (1990).

${ }^{4}$ Y. Cohen and E. L. Thomas, Macromolecules 21, 433 (1988).

${ }^{5}$ P. M. Jelf and N. A. Fleck, J. Compos. Mater. 26, 2706 (1992).

${ }^{6}$ L. D. Landau and E. M. Lifshitz, Theory of Elasticity (Pergamon, Oxford, 1986).

${ }^{7}$ T. Odijk, Macromolecules 28, 7016 (1995).

${ }^{8}$ T. Odijk, J. Chem. Phys. 105, 1270 (1996).
${ }^{9}$ G. W. Brodland, and R. J. Gordon, J. Biomech. Eng. 112, 319 (1990).

${ }^{10}$ P. A. Janmey, Curr. Opin. Cell Biol. 2, 4 (1991).

${ }^{11}$ D. E. Ingber, J. Cell. Sci. 104, 613 (1993).

${ }^{12}$ For recent qualitative remarks on this problem, see K. Kroy and E. Frey, Phys. Rev. Lett. 77, 306 (1996).

${ }^{13}$ H. Yamakawa, Modern Theory of Polymer Solutions (Harper \& Row, New York, 1971).

${ }^{14}$ T. Odijk, Macromolecules 19, 2313 (1986).

${ }^{15}$ J. Shimada and H. Yamakawa, Macromolecules 17, 689 (1984).

${ }^{16}$ T. Odijk, Macromolecules 16, 1340 (1983).

${ }^{17}$ S. R. Swanson, Trans. ASME 114, 8 (1992).

${ }^{18}$ S. Jain and D. R. Nelson, Macromolecules 29, 8523 (1995).

${ }^{19}$ G. Wegner, Macromol. Symp. 101, 257 (1996).

${ }^{20}$ T. Odijk, Europhys. Lett. 24, 177 (1993).

${ }^{21}$ K. Kassapidou and J. R. C. van der Maarel, Eur. J. Phys. B (in press).

${ }^{22}$ P. G. de Gennes, in Polymer Liquid Crystals, edited by A. Ciferri, W. R. Krigbaum, and R. B. Meyer (Academic, New York, 1982). 